\title{
Do Exercise and Be Mentally Strong
}

\author{
Bick C. Chow ${ }^{*}$ \\ Editor-in-Chief; \\ Department of Sports, Physical Education and Health, Hong Kong Baptist University, Hong Kong
}

Received: March 26, 2021

Accepted: March 26, 2021

Published online: April 30, 2021

Keywords:

COVID-19

Exercise

Physical Health
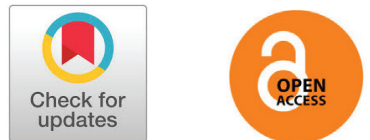

In the last year, we have witnessed an unprecedented pandemic affecting people's livelihoods and health worldwide. Certainly many people have experienced stress and anxiety arising from enforced social isolation during regional lock-down, quarantine or work-from-home mode. Amidst these negative effects affecting people's health, we have seen an increase in sales of home fitness equipment and more people getting into countryside for short hiking trips. These efforts are to undo the adverse effect upon people's physical health due to home confinement. Indeed, the concept of "wellness" has encompassed the intricate links among body, mind, and spirit (World Health Organization's Constitution to health, https://www.who.int/about/who-we-are/constitution). Better physical health often brings along positive mental health. Health and exercise science journals have published plenty of commentary, qualitative and experimental studies focusing on COVID-19 and they have presented this key message: importance of habitual physical activity and exercise as protective measure on COVID-19.

Being editor-in-chief of AJK, I would hope to see more publications including intervention studies illustrating effects of physical exercises on all areas related to kinesiology. The message on the importance of "Physical Activity and Exercise" in all phases of human's lifecycle should have wide dissemination so that more people will act on it.

*Correspondence: Bick C. Chow, Department of Sports, Physical Education and Health, Hong Kong Baptist University, Kowloon Tong, Hong Kong; Email: bchow@hkbu.edu.hk 